\title{
System for Automatic Registration of Remote Sensing Images
}

\author{
Dmitry Fedorov, Leila M.G. Fonseca \\ Division of Image Processing \\ National Institute for Space Research \\ 12.227-010 São José dos Campos, Brazil \\ e-mail: fedorov@dpi.inpe.br; leila@dpi.inpe.br \\ Charles Kenney, B.S. Manjunath \\ Department of Electrical and Computer Engineering \\ University of California, Santa Barbara, CA, USA. \\ e-mail: kenney@ece.ucsb.edu; manj@ece.ucsb.edu
}

\begin{abstract}
Image registration is the process of matching two images so that corresponding coordinate points in the two images correspond to the same physical region of the scene being imaged. It's an important operation needed in remote sensing that basically involves the identification of many control points. As the manual identification of control points may be time-consuming and tedious, automated techniques have been developed. This paper describes a system for automatic registration of satellite images under development at the Division of Image Processing (National Institute for Space Research - INPE) and the Vision Lab (Electrical \& Computer Engineering department, UCSB). The system provides tools that allow automatic registration and mosaic of remote sensing images. It is designed to accept different types of data (TM, SPOT, JERS, etc.) and information provided by the user that are used to speed up the processing or avoid mismatched control points. A statistical procedure is used to characterize good and bad registrations. Based on this "good fit-bad fit" statistical testing the user can stop or modify the parameters and continue the processing.

Extensive algorithm tests have been performed by registering optical, radar, multi-sensor, high-resolution images and video sequences. We have included very difficult image registration examples in order to show the strengths and limits of our approach. We developed a registration system online demo (http://nayana.ece.ucsb.edu/registration) that contains several examples that can be executed using web browser.
\end{abstract}

\section{INTRODUCTION}

The basic description of the point matching algorithm is presented in this section. The algorithm consists of a twostep procedure. In the first step, points in the first image are tentatively matched with points in the second image. This preliminary matching is accomplished by matching features at each tie point in the first image with features at each tie point in the second image. These features are extracted using optical flow [2] [3]. To overcome rotation effects, we have taken windows about each tie point that have been rotated so that their central gradient points downward. The initial feature matching can be used to eliminate points from both images that have no corresponding points. At the same time an initial corresponding points set (control points) in the two images is obtained. The second step refines the initial control points set by using a purely geometric matching procedure in which the location of points and their relationship to each other determine the matching. The geometric matching procedure is similar to the RANSAC algorithm [4]. Because the first and second steps are based on different matching criteria they provide a safeguard against mismatched points. Moreover the preliminary matching in the first step reduces the computational burden of the second step, resulting in a fast combined matching algorithm.

Once the tie point matching has been computed we need to verify its consistency. It is achieved using three independent tests. In the first two tests, a statistical procedure is used to characterize good and bad registrations. The computed registration is then compared with both good and bad fit estimation in order to determine its acceptability. This "good fit-bad fit" statistical testing is supplemented with a bootstrap parameter variation test in which control point subsets are used to re-compute the transformation parameters. Large parameter variation over the subsets indicates the presence of mismatched tie points. These three consistency-checking tests provide a powerful mechanism to detect improper registrations.

Extensive algorithm tests have been performed by registering optical, radar, multi-sensor, high resolution images and video sequences. We have included very difficult image registration examples in order to show the strengths and limits of our approach. We developed a registration system online demo [5] that contains several examples that can be executed using web browser.

\section{SYSTEM DESCRIPTION}

The developed registration system is a full featured application intended for beginners and advanced users. Registration may be achieved by one simple click or may 
be controlled by several parameters. System contains toolboxes what increase the registration strength using users knowledge. There's input images pre-processing what can change image resolution, select a specific band, enhance histogram, etc. Registration can be done through selected by user rectangles instead of using whole images. This is useful in cases with dense cloudiness, multi-temporal ocean shots, etc. Tie points may be edited in powerful embedded editor or exported to external applications.

Mosaic procedure contains several useful features like: mosaic both images into separate channels for match verification, equalize the images using simple mean and standard deviation comparation of the overlapping area, etc. System accepts most popular image formats (BMP, GIF, JPEG, PNG, RAW, TIFF, GeoTIFF, etc.) and some of mosaic results can be saved as GeoTiff images.

In order to simplify image viewing and handling a universal viewer was developed. It provides basic read/write and preview operations, such as: load file, save file, change zoom, image information, full screen preview, clipboard handling, etc. Visualization module also contains a layer to handle special information like tie points, rectangles, etc.

The system is developed with multi-platform use in mind, it have been compiled and used under Solaris, Linux and Windows.

The system is separated in two logical parts. The first part is the main driver for registration and mosaicing. It is written using common $\mathrm{C}++$ and Trolltech Qt [6] library for image representation, which provides better platform independence (Unix, Windows, Mac) and also independence from user interaction layer.

Registration procedure needs only one parameter, which specifies the number of tie points to start with. This parameter is only modified in more complicated registration cases (images with clouds, severe temporal changes, very different sensors) and decreases registration speed exponentially. However, reasonable number of tie points (128) returns almost instant result.

There are two user interaction layers. The first one is a command line module intended for calls from other systems, for example, it's used by CGI demo script.

The second is a GUI module written on $\mathrm{C}++$ using Trolltech Qt library. We developed or modified several image file handlers for Qt, such as: RAW reader/writer, JPEG encoder/decoder using Independent JPEG Group library and TIFF encoder/decoder using libTIFF from SGI and GeoTIFF encoder.

\section{REGISTRATION EXAMPLES}

In order to test the system and demonstrate its feasibility for different types of images some preliminary results are presented in this section.
Figure 1 shows the registration of two images from the urban area of São Paulo. A SPOT image, band 3 was reduced to $30 \mathrm{~m}$ pixel size and registered with LandsatTM5 image, band 4.

Figure 2 shows the registration of Amazon region images taken from TM5 sensor, band 5, in different dates.

Figure 3 shows the registration of Amazon tropical forest images taken from TM5 sensor, band 5, in different dates. These images represent high cloud cover and were successfully registered with 512 points. Other experiments with SPOT and TM images from urban, forest and agricultural areas were performed and in all cases we obtained very encouraging results.

Figure 4 shows the registration of $\mathrm{B} / \mathrm{W}$ aerial images of Lompoc city, California, in different dates.

Figure 5 and Figure 6 shows the registration of the old aerial B/W sequence of Goleta, California.

Figure 6 shows the registration of Amazon region images taken from JERS-1, in different dates.

Figure 7 shows a mosaic of an aerial video sequence taken from the Amazon region.

Finally, Figure 8 shows the registration system graphical user interface in windows.

\section{REFERENCES}

[1] Fonseca, L.M.G, Manjunath, B.S., "Registration techniques for multisensor remotely sensed imagery". PE\&RS, Vol. 62, No. 9, Sept. 1996, pp. 1049-1056.

[2] Fonseca, L M.G, Hewer, G., Kenney, C., and Manjunath, B.S., "Registration and Fusion of Multispectral Images Using a New Control Point Assessment Method Derived from Optical Flow Ideas", Proc. SPIE, Vol. 3717, pp.104-111, April 1999, Orlando, FLA.

[3] Fonseca, L M.G, Kenney, C., "Control Point Assessment for Image Registration”, Proc. XII Brazilian Symposium on Computer Graphics and Image Processing, Campinas, Brazil, October 1999, pp. 125-132.

[4] Hartley R., Zisserman A., "Multiple View Geometry in Computer Vision", Cambridge University Press, 2000.

[5] Fedorov D. V., Kenney C., Manjunath B.S., Fonseca L. M. G., "Online registration demo". <http://nayana.ece.ucsb.edu/registration/>, 2001-2002.

[6] Trolltech AS, <http://www.trolltech.com> 

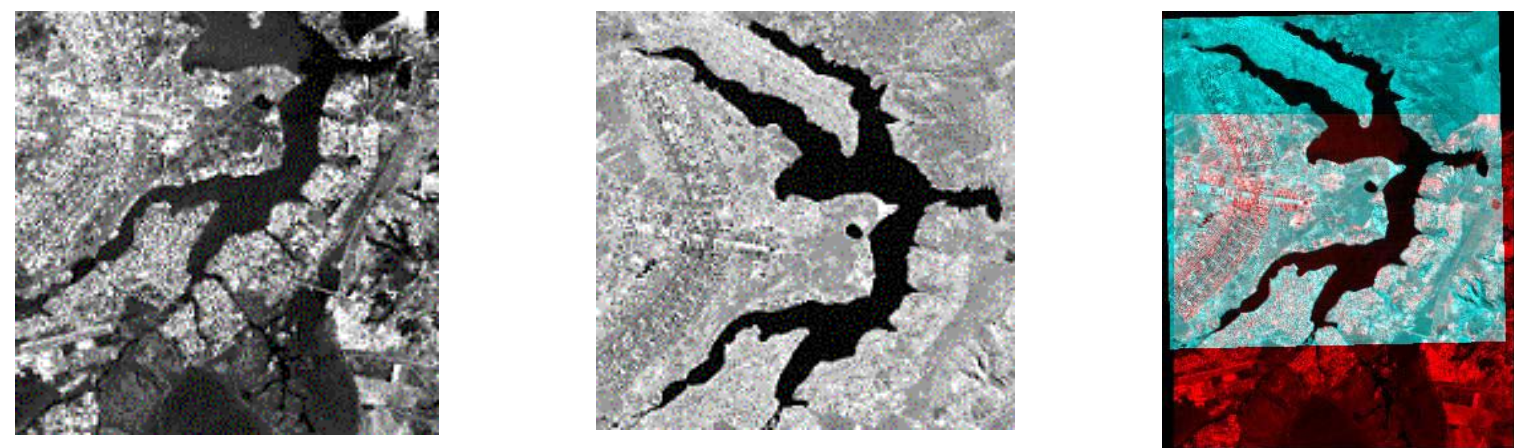

Figure 1: Urban, SPOT band 3 (08/08/95) + TM band 4 (06/07/94), 256x256, Brasilia, Brazil. Good registration with 128 points.
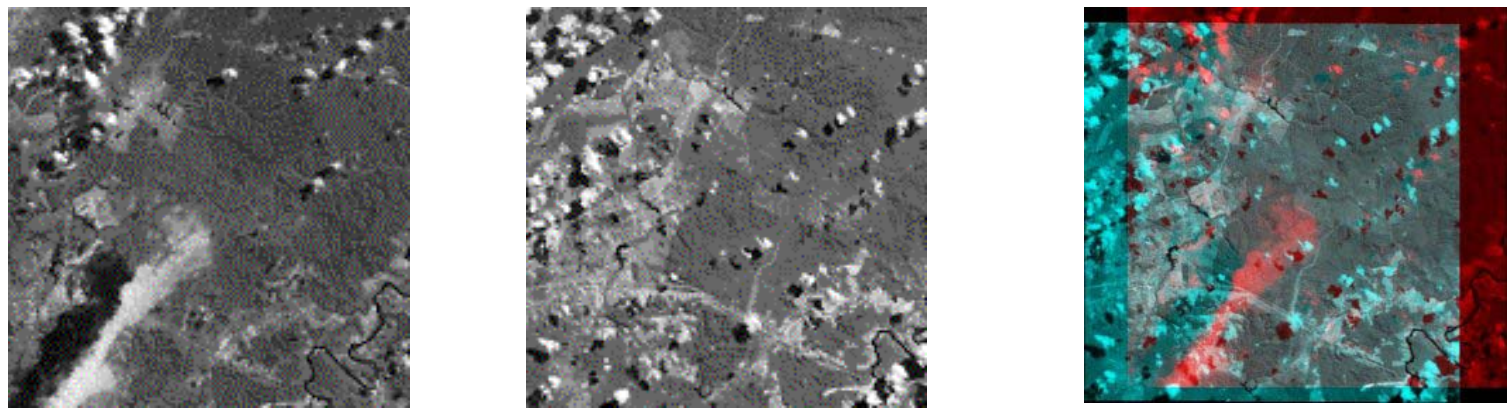

Figure 3: Tropical forest, Landsat-TM band 5, (August 29, 1993, and August 7, 1995), 512x512, Tapajós, Amazon, Brazil. Good registration with 512 points.
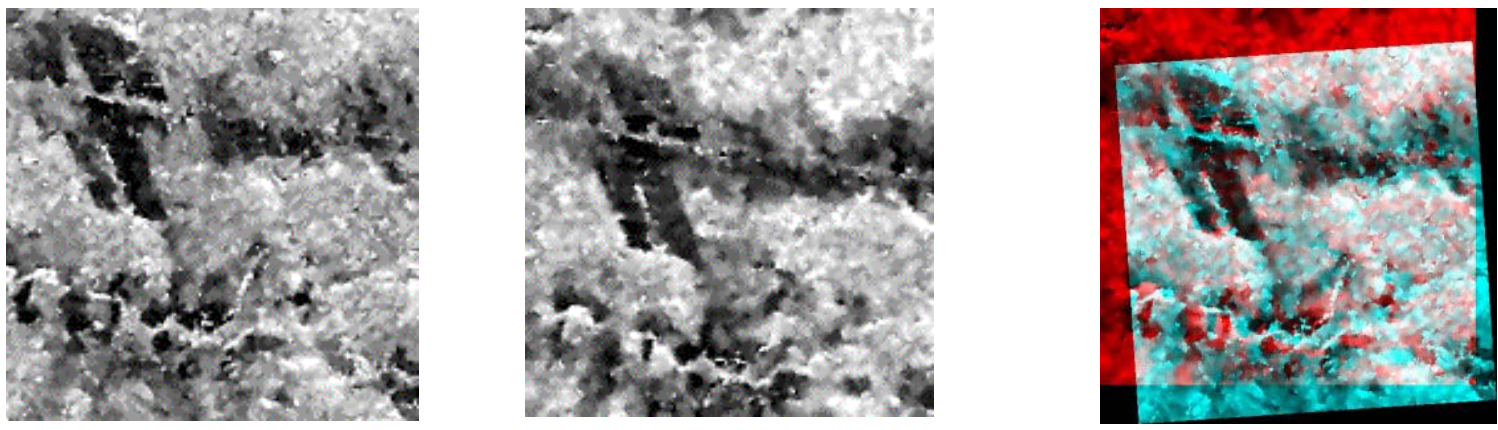

Figure 6: Radar, JERS-1, (10/10/95) + (08/13/96), 512x512, Amazon, Brazil. Good registration: 128 points.

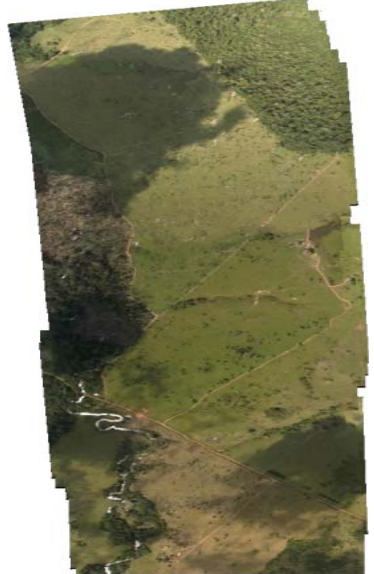

Figure 7: Aerial video sequence mosaic, 720x480, Amazon, Brazil.

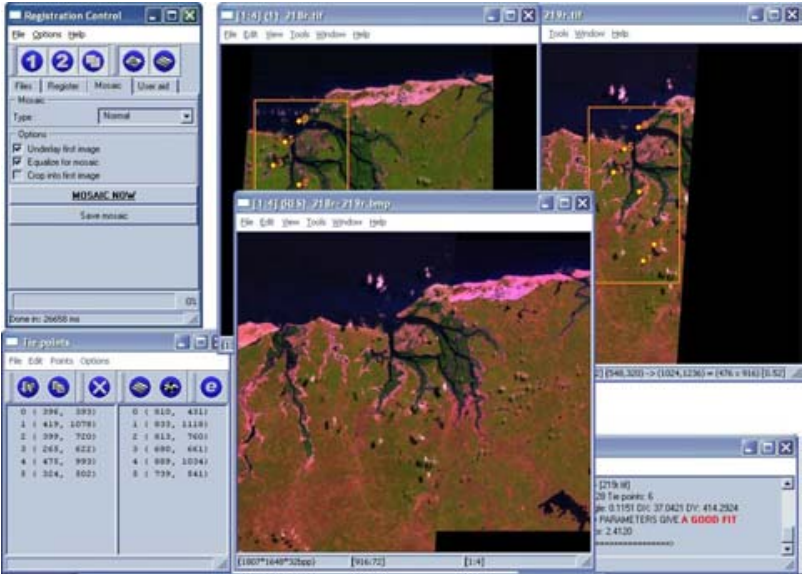

Figure 8: System graphical user interface in windows. 